\title{
Is it Possible to Mathematically Predict the outcomes of Treatment of Abdominal Inflammation?
}

\author{
Sławomir Jabłoński*1, Sylwia Kustalik ${ }^{1}$, Magdalena Drzewiecka-Jędrzejczyk², Piotr Misiak ${ }^{1}$, Szymon \\ Wcisło $^{1}$ and Marcin Kozakiewicz ${ }^{3}$
}

${ }^{1}$ Department of Thoracic Surgery, General and Oncological Surgery, Medical University of Lodz, Poland

${ }^{2}$ Department of Endocrine, General and Oncological Surgery, Medical University of Lodz, Poland

${ }^{3}$ Department of Maxillofacial Surgery, Medical University of Lodz, Poland

Received: June 04, 2018; Published: June 20, 2018

*Corresponding author: Sławomir Jabłoński, Department of Thoracic Surgery, General and Oncological Surgery, Medical University of Lodz, $90-549$ Łódź, 113 Żeromskiego St., 17 Daleka St., 93-348 Łódź, Poland

\author{
Abstract \\ Aim: The aim of this study was to assess whether the statistical determination of the outcomes of treatment of abdominal inflammation \\ is possible.
}

Materials and Methods: The sample size in this study consisted of 49 patients who underwent surgery due to abdominal inflammatory states. Data for six biochemical and two clinical parameters were collected for the purpose of constructing a prognostic scale designed by the authors.

Results: The multi-dimensional space that resulted from the data collected was reduced to a three-dimensional model by analysis of 3 factors: inflammation, proteinic status and general risk. Firstly, all cases were categorized based on their respective composite Recovery Prediction Factor (SNC=62\%, SPC=55\%). Secondly, it was determined that two cases could not be categorized due to their dichotomic division based on factors 1, 2 and 3 (SNC=79\%, SPC=95\%). Lastly, based on its median origin dichotomic division ( $\mathrm{SNC}=72 \%$, SPC=84\%), one case could not be categorized.

Conclusion: It is possible to predict the outcomes of the treatment of abdominal inflammation, based on statistical factor analysis and a factor average prediction engine. However, some degree of uncertainty must be taken into account with respect to the probability that a given outcome may unfold in a given patient.

Keywords: Intra-Abdominal Infections; Prediction Method; Inflammatory Status; Proteinic Status; Recovery Prediction Factor; Sepsis

Abbreviations: HGB: Hemoglobin Level; HCT: Hematocrit; WBC: White Blood Cell Count; Na: Serum Sodium; K: Potassium Level; CRP-C: Reactive Protein; PCT: Procalcitonin; AM Risk Calculator: Acute Mediastinitis Risk Calculator; SNC: Sensitivity Coefficient; SPC: Specifity Coefficient; ICED: Index of Coexistent Diseases; NRI - Nutritional Risk Index; GNRI: Geriatric Nutritional Risk Index; PINI: Prognostic Inflammatory and Nutritional Index; ASA: American Society of Anesthesiologists Physical Status; LOD: Logistic Organ Dysfunction system; RPF: Recovery Prediction Factor

\section{Background}

Intra-abdominal infections are characterized by both a high rate of incidence and mortality in patients treated surgically. It is believed that in the first decade of the $21^{\text {st }}$ century, approximately $20 \%$ of patients following major visceral surgery developed severe sepsis. After pneumonia, it is the second most common cause of infectious morbidity and mortality in intensive care units [1]. Despite advancements in surgical techniques, intensive therapy or new generations of antibiotics and other pharmaceutical compounds, the results of treatment of abdominal sepsis are poor. What follows are prolonged hospitalization and increased costs of treatment. This is predominantly the concern of patients receiving treatment in intensive therapy wards requiring further surgical intervention. In the CIAO conducted by Sartelli et al, the overall mortality rate in patients with complicated intra-abdominal infections was 7,7\% (166/2152) [2]. However, if patients with severe sepsis or septic shock on admission to hospital are taken into account, the mortality rate increased from between $10 \%$ and $42,3 \%[3,4]$.

Studies have been ongoing for many years in the search for clinical, biochemical and immunological parameters to determine 
early, statistically significant predictive and independent risk factors for developing severe sepsis. This data may significantly influence the treatment of choice, be it surgical, a broadening of conservative treatment or the initiation of therapy with antibiotics. Nevertheless, the most serious problem in these clinical studies is the analysis of a large heterogeneous group of patients based on a large number of causes of sepsis: pneumonia, peritonitis, pancreatitis, soft tissue infection or burn injury. Regardless of the differences in source of the infection, large differentiation is observed between aspects such as: age, sex, pathogenic spectrum, underlying comorbidity, etc. [5]. In order to prevent the disease course from influencing the prognosis, many prognostic scales based on many clinical and diagnostic data have been developed. Among the most commonly known and utilized such tools are the Charlson Co-Morbidity Index [6], the Davies (Stokes) Score [7] and the Index of Coexistent Diseases (ICED) [8].

Other prognostic scales used to score the severity of postoperative complications include the Nutritional Risk Index (NRI) [9], the Geriatric Nutritional Risk Index (GNRI) [10], the Prognostic Inflammatory and Nutritional Index (PINI) [11], the American Society of Anesthesiologists (ASA) Physical Status [12], and the Logistic Organ Dysfunction (LOD) System [13]. The authors of that study had previously developed a unique prognostic scale of their own for use in the analysis of patients suffering from acute mediastinitis, called the AM Risk Calculator. It was found to have strong diagnostic value (SNC=90\%, SPC=64\%) [14]. In the group of patients with iatrogenic acute mediastinitis, its value was even higher (SNC=100\%, SPC=69,2\%) [15]. In this study, the authors assessed the viability of the AM Risk Calculator in the prognosis of patients with intra-abdominal infections as well as the design and implementation of appropriate modifications so as to better examine septic complications of this etiology.

\section{Purpose}

The aim of this study was to evaluate if the outcomes of abdominal inflammation treatment can be predicted based on the prognostic scale designed by the authors, specifically that of patients with acute mediastinitis.

\section{Material and Methods}

A total of 49 patients were qualified for this study, each having received emergency surgical treatment at the Department of Thoracic Surgery, General and Oncological Surgery at University Clinical Hospital No.2 -Military Memorial Medical Academy Central Veterans' Hospital, Medical University of Lodz, Poland, due to inflammatory states of the abdominal cavity, such as: perforation or stenosis of the gastric tract or intraperitoneal abscess. This study was approved by the Bioethics Commission of the Medical University of Lodz (nr.RNN/481/12/KB). The following clinical risk factors were evaluated: age, gender, etiology, number of comorbidities, type of surgical procedure, the kind and number of postoperative complications and the total time of hospitalization. The association between mortality rate and the selected biochemical risk factors was then assessed by analyzing the following parameters: hemoglobin level (HGB), hematocrit (HCT), red blood cell count, white blood cell count (WBC), platelet count, as well as serum sodium $(\mathrm{Na})$, potassium $(\mathrm{K})$, protein, albumin, C-Reactive protein (CRP), and procalcitonin (PCT) levels.

According to previous studies $[14,15]$, factor analysis was used to determine the risk factors for patients with intra-abdominal infections. Designed with the purpose of predicting possible prognoses, the "AIM Risk Calculator" takes into account three factors which affected the final result: Factor 1: inflammatory status (WBC, CRP and PCT), Factor 2: proteinic status (serum level protein, albumin and HGB) and Factor 3: general risk (age and number of coexisting diseases). Unfortunately, the utilization of the same eight parameters, as used with respect to acute mediastinitis, deteriorated the prognostic predictive power. The principle cause of the variability in the original data was achieved following the exclusion of the WBC parameter. The coefficients of sensitivity and specificity were calculated for the factors so as to determine the prediction power of the proposed method.

The method was designed for the prediction of recovery, thus the result of the test is positive $[\mathrm{P}]$ when the test predicts that recovery is likely; negative $[\mathrm{N}]$ when recovery is unlikely, i.e. the death of the patient. Respectively, the result of the test was true [T] when the test predicts a likely recovery and the clinically evident result was "recovery"; the result of the test being negative [N] when the test did not predict a likely recovery. Subsequently, the following data was generated: TP - patient recovered as predicted, TN - patient death as predicted, FP - patient death predicted as "recovery" and FN - patient recovery predicted as "death". Based on the above definitions, the following sensitivity and specificity coefficients equation were proposed:

Sensitivity coefficient:

$$
S N C=T P /(T P+F N) \times 100 \%
$$

Specificity coefficient:

$$
S P C=T N /(T N+F P) \times 100 \%
$$

Statistical analysis was performed using the software application Statgraphics Centurion XVI (Statpoint, Technologies inc. USA).

\section{Results}

The inflammatory states of the abdominal cavity of the 49 patients analyzed in this study were: perforation of the gastric tract, of those 20 were due to peptic and duodenal ulcers, three due to gastric cancer, and seven due to colon cancer; stenosis of the gastric tract [16], of those 10 were due to a tumor of the colon and six were due to a state of inflammation; intra-abdominal abscess [3], 2 of which were due to adnexitis and 1 due to diverticulitis. The patients ranged in age from 22 to 91 years with a mean age of 64, 9 years (median 67, 5 years). The study group was comprised of 20 men and 29 women. All patients underwent surgical intervention; 14 of whom died postoperatively. The combined death rate was $28,57 \%$ (30\% among the men and 27, 59\% among the women). It was determined that patient age had a significant impact on his or her ultimate prognosis. Among the patients in the [death] 
group, the mean age was 76 years and the media age was 79,5 . In the [recovery] group, the mean and median ages were 57,5 and 61 years, respectively. The P-value was 0,000939.

The number of comorbidities ranged from 0 to 10 . The mean was higher among those patients who died postoperatively: 4, 45 (median 4, 0) compared with the [recovery] group: 2, 63 (median 2, 0 ). The duration of hospitalization ranged from 3 to 31 days; mean 11, 14 (median 10). The preoperative biochemical parameters were as follows: CRP average: 258, 726 (median: 240, 8), PCT average: 17, 218 (median: 9, 5), HGB average: 12, 358 (median: 12, 45), WBC average: 13, 5292 (median: 12, 15), protein average: 49, 45 (median: 50, 8) and albumin average: 25, 16 (median: 23, 6). Differences in these biochemical parameters were observed between those pertaining to patients who recovered versus those who died. The final values of the inflammatory markers were higher among those who died, with the exception of WBC. The level of CRP in the [death] group had a mean of 301, 8 (median: 230, 01 ); in the [recovery] group the mean was 230, 01 (median: 197, 65). The value of CRP was determined to be statistically significant: P-value $=0,0135$. The level of PCT in the [death] group was higher as well, mean 30, 38 (median: 25, 35), than in the [recovery] group, mean 8, 44 (median: 6, 75). The PCT parameter was also found to be statistically significant: P-value $=0,00000393987$. The preoperative level of WBC was found to be statistically insignificant for the purpose of prognostic prediction. The following values of WBC were determined: in the [death] group, mean 13, 47 (median 12, 95); in the [recovery] group, mean 13, 57 (median: 11, 5) and P-value $=0,9642$.

In the case of those biochemical parameters consolidated by the authors into the single the proteinic factor, their values were lower among the patients who died. The level of proteins in the [death] group was mean 43,295 (median: 43, 85), in the [recovery] group, mean 53, 68 (median: 54,9). The authors confirmed that the low protein level indicated a strong statistical significance for the prediction of the development of unfavorable treatment outcomes: P-value $=0,0007$. A co-relation was observed between the values of the albumin factor: in the [death] group the mean was 21, 97 (median: 21,65); in the [recovery] group, mean: 27,35 (median: $28,8)$. In this case the value was statistically significant: P-value $=$ 0,0033 . No corelation was determined between the risk of death and the preoperative HGB value: in the [death] group, mean 11, 62 (median: 12, 55); in the [recovery] group, mean 12,85 (median: 12,4); no statistical significance: P-value $=0,289$.

The values of the eight parameters (age, comorbidities, albumins, protein, HGB, WBC, CRP and PCT) were grouped into three factors of the prognostic scale as per the design of the authors. They were used to determine the prognoses of patients with sepsis of the abdominal cavity. Together the 3 factors accounted for $79 \%$ of the variability in the original data (Fig. 1). The factor loading matrix was rotated to improve the interpretation of the factors of the prognostic scale, calculated as below:

a) Factor $1=0,237986 * \mathrm{HGB}+0,348462 * \mathrm{Age}+$ 0,857876*CRP_pre + 0,871758*PCT_pre $+0,370096^{*}$ Co-exist_ diseases - 0,154912*Proteins - 0,189613*Albumins b) Factor $2=0,121001^{*} \mathrm{HGB}-0,0696146^{*}$ Age 0,182683*CRP_pre - 0,207829*PCT_pre - 0,0952214*Co-exist_ diseases $+0,928589^{*}$ Proteins $*+0,931407 *$ Albumins

c) Factor $3=-0,826901^{*} \mathrm{HGB}+0,749317^{*}$ Age + $0,0805403^{*}$ CRP_pre $+0,151458^{*}$ PCT_pre $+0,715845^{*}$ Coexist_diseases $-0,136637^{*}$ Proteins $-0,0635118^{*}$ Albumins

Due to factor loading (presented in bold) the factors were established as follows: Factor1 as inflammation status; Factor2 as proteinic status and Factor3 as general risk. The mean of all factors was close to the value of 0 (Table 1), along with their high standard deviations and normal distributions. It was impossible to derive a simple predictive range of each factor due to their overlapping one another.

Table 1: Statistical summary of the factors calculated.

\begin{tabular}{|c|c|c|c|}
\hline & $\begin{array}{c}\text { Inflammatory } \\
\text { status F1 }\end{array}$ & $\begin{array}{c}\text { Proteinic status } \\
\text { F2 }\end{array}$ & General risk F3 \\
\hline Mean & 0,000000346939 & $-0,000000320408$ & $-0,000000173469$ \\
\hline Median & $-0,414016$ & $-0,301148$ & $-0,0748673$ \\
\hline Std. Dev. & 2,07188 & 2,01161 & 1,94881 \\
\hline Minimum & $-3,39467$ & $-3,74289$ & $-4,71265$ \\
\hline Maximum & 5,72188 & 4,66413 & 3,8678 \\
\hline Range & 9,11655 & 8,40702 & 8,58045 \\
\hline $\begin{array}{c}\text { Std. } \\
\text { Skewness }\end{array}$ & 1,96696 & 0,610406 & 0,0198361 \\
\hline $\begin{array}{c}\text { Std. } \\
\text { Kurtosis }\end{array}$ & 0,198719 & $-1,09831$ & $-0,326157$ \\
\hline
\end{tabular}

For this reason, the authors defined a composite Recovery Prediction Factor (RPF). The composition of this formula was intended to improve the separation of the factors for the purpose of deriving the recovery prediction pattern.

$R P F=\log \left[\left(\left[\sigma_{-} F 2\left(F 2-\mu_{-} F 2\right)\right)^{\wedge} 93 /\left(\left[\sigma_{-} F 1\left(F 1-\mu_{-} F 1\right)\right)^{\wedge} 93+\left[\sigma_{-} F\left(F 3-\mu_{-} F 3\right) \mathbf{I}^{\wedge} 93\right)\right)^{\wedge} 2\right]\right.$

Where:

RPF - Recovery Prediction Factor

F1, F2, F3 -values of Factor1, Factor2, Factor3, respectively

$\mu \mathrm{F} 1, \mu \mathrm{F} 2, \mu \mathrm{F} 3$-averages of Factor1, Factor2, Factor3, respectively

$\sigma \mathrm{F} 1, \sigma \mathrm{F} 2, \sigma \mathrm{F} 3$-standard deviations of Factor1, Factor2, Factor3, respectively

Table 2: Distribution of the composite Recovery Prediction Factor (RPF) of the total sample size and of both subgroups.

\begin{tabular}{|c|c|c|c|}
\hline & $\mathbf{R P F}$ & $\mathbf{R P F}_{\text {Death }}$ & $\mathbf{R P F}_{\text {Recovery }}$ \\
\hline $\begin{array}{c}\text { Number of } \\
\text { cases }\end{array}$ & 49 & 20 & 29 \\
\hline Average & $-29,7736$ & $-55,6169$ & $-11,9507$ \\
\hline Median & $-29,8881$ & $-45,894$ & $-29,4505$ \\
\hline $\begin{array}{c}\text { Standard } \\
\text { deviation }\end{array}$ & 77,1324 & 70,5777 & 77,5424 \\
\hline Minimum & $-251,277$ & $-251,277$ & $-157,315$ \\
\hline Maximum & 222,048 & 45,7289 & 222,048 \\
\hline
\end{tabular}




\begin{tabular}{|c|c|c|c|}
\hline Range & 473,325 & 297,006 & 379,363 \\
\hline Stnd. skewness & 0,215461 & $-2,1938$ & 1,32642 \\
\hline Stnd. kurtosis & 3,17332 & 1,70352 & 2,08323 \\
\hline
\end{tabular}

The sum of the significant negative factors was used as the denominator and the positively determined factor was the numerator. The composite factor served as the recovery predictor. The use of average value subtraction and division by the standard deviation stabilized the factor values in one another's separate regions. The high exponentiation increased the separation up to the limit of statistical significance. The logarithm normalized the distribution of RPF and the square exponentiation ensured the derivation of a positive number prior to logarithmic calculation (Table 2).

It can now be observed that the significant separation $(\mathrm{F}=4.03$, $\mathrm{p}=0.0504$ ) was reached between the RPF values depending on patient outcome. The RPF boundary value can easily be established between RPF Death and RPF Recovery in point 34. Recovery can be predicted as likely in cases where the RPF is greater or equal to 34 and predicted as unlikely when the RPF is less than 34 . The coefficients of sensitivity and specificity were determined to be $\mathrm{SNC}=62 \%$ and $\mathrm{SPC}=55 \%$ (Table 3). Next, to determine the prediction method, the authors referred to the original Factors 1, 2 and 3. Due to the normal distribution of the values of these factors, their mean indicated the presence of causality for the dichotomic division of their values. For inflammatory status: $\mathrm{SNC}=85 \%$ and $\mathrm{SPC}=73 \%$; for proteinic status: $\mathrm{SNC}=62 \%$ and $\mathrm{SPC}=85 \%$; for general risk factor $\mathrm{SNC}=69 \%$ and $\mathrm{SPC}=65 \%$. These partial predictions were then re-evaluated. Given that two of the three factors indicated the same outcome, the final prediction was derived from the average of this outcome. The coefficients of sensitivity and specificity were then determined as $\mathrm{SNC}=79 \%$ and $\mathrm{SPC}=95 \%$. However, it was impossible to categorize two cases in this study due to all of their factors having predicted another result, i.e. FN, TN and FP in case 20, and FP, FN and TP in case 47 (Table 3).

Lastly, the factor values were divided by their median for the purpose of predicting the outcome of treatment. As the values of Factors 1 and 3 was higher than their medians (-0.41 for Inflammatory Status and -0.07 for General Risk), their prediction was established as death. In contrast, values less than or equal to their corresponding medians would predict a recovery. As the value calculated for Factor 2 was greater or equal to its median, (-0.3 for Proteinic Status), the prediction was recovery. Patient death was predicted in the event where the value of Factor 2 value was less than its median. Coefficients of sensitivity and specificity were calculated for each factor: Factor 1 (SNC=73\%, SPC=84\%); Factor 2 (SNC=69\%, SPC=75\%); Factor 3 (SNC=69\%, SPC=75\%). The classification of the prevalence tests [TP, TN, FP, FN] was determined so as to establish the overall prognostic power of this technique with respect to the cases of abdominal inflammation investigated: $\mathrm{SNC}=72 \%, \mathrm{SPC}=84 \%$ (Table 3). There remains the issue of the impossibility to categorize Case 5 due to divergent indications of all of its factors, i.e. TP, FP and TN.

Table 3: Summary of data used to predict the treatment outcome based on three techniques of prediction: RPF, factor mean and factor median.

\begin{tabular}{|c|c|c|c|c|c|c|c|c|c|c|c|c|c|c|}
\hline \multirow[t]{2}{*}{ 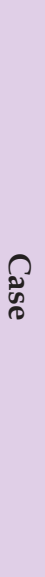 } & \multirow{2}{*}{ 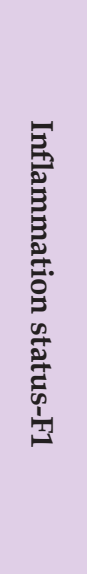 } & \multirow{2}{*}{ 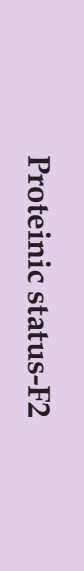 } & \multirow{2}{*}{ 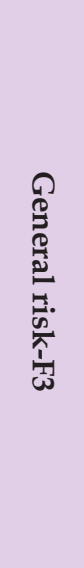 } & \multirow{2}{*}{ 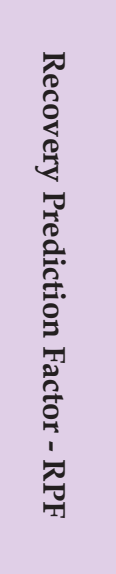 } & \multirow{2}{*}{ 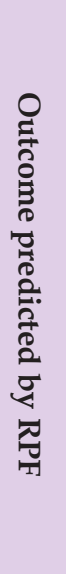 } & \multicolumn{4}{|c|}{ 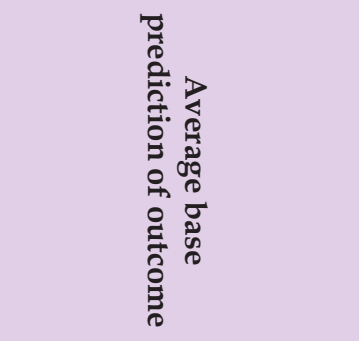 } & \multicolumn{4}{|c|}{ 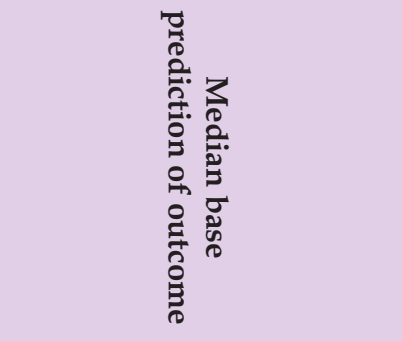 } & \multirow{2}{*}{ 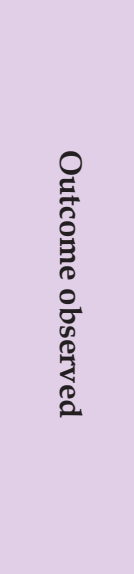 } \\
\hline & & & & & & 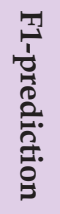 & 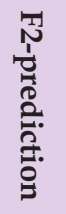 & 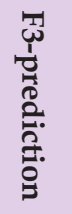 & 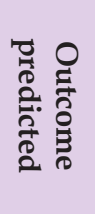 & 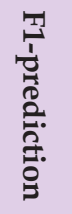 & 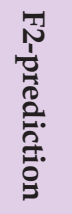 & 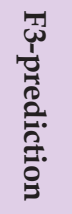 & 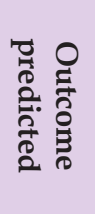 & \\
\hline 1 & 0,29 & 4,66 & $-0,07$ & 222,05 & $\mathrm{TP}$ & FP & $\mathrm{TP}$ & $\mathrm{TP}$ & $\mathrm{TP}$ & $\mathrm{FN}$ & $\mathrm{TP}$ & $\mathrm{TP}$ & $\mathrm{TP}$ & Recovery \\
\hline 2 & 0,75 & 0,24 & 0,19 & $-93,00$ & $\mathrm{TN}$ & $\mathrm{TN}$ & $\mathrm{FP}$ & $\mathrm{TN}$ & $\mathrm{TN}$ & $\mathrm{TN}$ & $\mathrm{FP}$ & $\mathrm{TN}$ & $\mathrm{TN}$ & Death \\
\hline 3 & 2,17 & $-0,44$ & 3,87 & $-173,51$ & $\mathrm{TN}$ & $\mathrm{TN}$ & $\mathrm{TN}$ & $\mathrm{TN}$ & $\mathrm{TN}$ & $\mathrm{TN}$ & $\mathrm{TN}$ & $\mathrm{TN}$ & $\mathrm{TN}$ & Death \\
\hline 4 & 1,28 & $-2,33$ & $-0,31$ & 45,73 & $\mathrm{FP}$ & $\mathrm{TN}$ & TN & $\mathrm{FP}$ & $\mathrm{TN}$ & $\mathrm{TN}$ & $\mathrm{TN}$ & $\mathrm{FP}$ & $\mathrm{TN}$ & Death \\
\hline 5 & $-1,16$ & 1,03 & 1,00 & $-11,43$ & FP & $\mathrm{TN}$ & $\mathrm{FP}$ & $\mathrm{TN}$ & $\mathrm{TN}$ & $\mathrm{TP}$ & $\mathrm{FP}$ & $\mathrm{TN}$ & $?$ & Death \\
\hline 6 & $-0,11$ & $-1,23$ & 0,42 & 89,61 & $\mathrm{TP}$ & $\mathrm{TP}$ & $\mathrm{FN}$ & FN & FN & FN & FN & FN & $\mathrm{FN}$ & Recovery \\
\hline 7 & 1,64 & $-0,89$ & 2,94 & $-94,04$ & $\mathrm{TN}$ & $\mathrm{TN}$ & $\mathrm{TN}$ & $\mathrm{TN}$ & $\mathrm{TN}$ & $\mathrm{TN}$ & $\mathrm{TN}$ & $\mathrm{TN}$ & $\mathrm{TN}$ & Death \\
\hline 8 & 0,68 & $-1,71$ & 1,58 & 8,84 & $\mathrm{FP}$ & TN & $\mathrm{TN}$ & $\mathrm{TN}$ & $\mathrm{TN}$ & $\mathrm{TN}$ & $\mathrm{TN}$ & $\mathrm{TN}$ & $\mathrm{TN}$ & Death \\
\hline 9 & 3,96 & $-3,53$ & 1,31 & $-11,88$ & FP & $\mathrm{TN}$ & $\mathrm{TN}$ & $\mathrm{TN}$ & $\mathrm{TN}$ & $\mathrm{TN}$ & $\mathrm{TN}$ & $\mathrm{TN}$ & $\mathrm{TN}$ & Death \\
\hline
\end{tabular}




\begin{tabular}{|c|c|c|c|c|c|c|c|c|c|c|c|c|c|c|}
\hline 10 & 0,90 & $-0,30$ & $-0,39$ & $-90,81$ & TN & TN & $\mathrm{TN}$ & FP & TN & TN & FP & FP & FP & Death \\
\hline 11 & $-0,41$ & $-2,32$ & $-1,18$ & 57,36 & $\mathrm{TP}$ & $\mathrm{TP}$ & FN & $\mathrm{TP}$ & $\mathrm{TP}$ & $\mathrm{TP}$ & FN & $\mathrm{TP}$ & $\mathrm{TP}$ & Recovery \\
\hline 12 & $-1,03$ & 1,20 & $-0,80$ & 10,24 & FP & FN & FP & FP & FP & FP & FP & FP & FP & Death \\
\hline 13 & $-0,12$ & $-0,76$ & 2,94 & $-107,16$ & $\mathrm{TN}$ & FN & $\mathrm{TN}$ & $\mathrm{TN}$ & $\mathrm{TN}$ & $\mathrm{TN}$ & $\mathrm{TN}$ & $\mathrm{TN}$ & TN & Death \\
\hline 14 & $-1,17$ & 2,31 & $-0,62$ & 52,42 & TP & TP & TP & $\mathrm{TP}$ & TP & TP & TP & TP & TP & Recovery \\
\hline 15 & $-0,51$ & 1,21 & $-1,98$ & $-37,21$ & FN & $\mathrm{TP}$ & $\mathrm{TP}$ & $\mathrm{TP}$ & $\mathrm{TP}$ & $\mathrm{TP}$ & $\mathrm{TP}$ & $\mathrm{TP}$ & $\mathrm{TP}$ & Recovery \\
\hline 16 & $-0,55$ & $-0,44$ & 0,66 & $-29,89$ & $\mathrm{TP}$ & ТP & FN & FN & FN & TP & FN & FN & FN & Recovery \\
\hline 17 & $-1,78$ & 2,45 & $-0,81$ & 23,34 & TP & TP & TP & TP & TP & TP & TP & TP & TP & Recovery \\
\hline 18 & 2,88 & $-2,44$ & $-0,12$ & $-15,85$ & FP & TN & $\mathrm{TN}$ & FP & $\mathrm{TN}$ & TN & $\mathrm{TN}$ & FP & TN & Death \\
\hline 19 & $-1,74$ & $-0,08$ & 1,00 & $-251,28$ & TN & FN & TN & TN & $\mathrm{TN}$ & FP & FP & TN & FP & Death \\
\hline 20 & $-1,20$ & $-0,56$ & $-0,04$ & $-64,46$ & TN & FN & $\mathrm{TN}$ & FP & $?$ & FP & $\mathrm{TN}$ & TN & TN & Death \\
\hline 21 & $-1,06$ & 0,93 & $-0,51$ & $-13,38$ & $\mathrm{TP}$ & $\mathrm{TP}$ & TP & $\mathrm{TP}$ & ТP & $\mathrm{TP}$ & TP & TP & $\mathrm{TP}$ & Recovery \\
\hline 22 & $-2,46$ & 1,69 & 0,17 & $-32,66$ & $\mathrm{TP}$ & $\mathrm{TP}$ & TP & $\mathrm{FN}$ & $\mathrm{TP}$ & TP & TP & FN & TP & Recovery \\
\hline 23 & 2,31 & $-2,02$ & $-1,56$ & $-13,33$ & FP & TN & TN & FP & TN & TN & TN & FP & TN & Death \\
\hline 24 & $-3,39$ & 1,65 & $-3,05$ & $-60,53$ & FN & $\mathrm{TP}$ & TP & $\mathrm{TP}$ & ТP & TP & TP & $\mathrm{TP}$ & $\mathrm{TP}$ & Recovery \\
\hline 25 & $-1,08$ & $-0,35$ & $-1,73$ & $-125,72$ & FN & $\mathrm{TP}$ & FN & TP & TP & $\mathrm{TP}$ & FN & TP & $\mathrm{TP}$ & Recovery \\
\hline 26 & $-2,14$ & 2,97 & $-4,71$ & $-34,83$ & FN & $\mathrm{TP}$ & TP & $\mathrm{TP}$ & $\mathrm{TP}$ & $\mathrm{TP}$ & $\mathrm{TP}$ & TP & TP & Recovery \\
\hline 27 & $-1,91$ & 2,57 & $-0,63$ & 21,81 & TP & ТP & TP & $\mathrm{TP}$ & TP & TP & TP & $\mathrm{TP}$ & TP & Recovery \\
\hline 28 & 1,05 & $-3,74$ & 3,47 & 8,73 & FP & TN & TN & $\mathrm{TN}$ & $\mathrm{TN}$ & TN & $\mathrm{TN}$ & TN & TN & Death \\
\hline 29 & 2,65 & $-0,64$ & 1,48 & $-117,70$ & FN & FP & FN & $\mathrm{FN}$ & FN & FN & FN & FN & FN & Recovery \\
\hline 30 & $-3,05$ & 3,27 & $-3,08$ & 3,32 & $\mathrm{TP}$ & TP & TP & TP & TP & TP & TP & TP & TP & Recovery \\
\hline 31 & $-2,46$ & 2,60 & $-3,68$ & $-25,42$ & ТP & $\mathrm{TP}$ & TP & $\mathrm{TP}$ & TP & TP & TP & ТP & $\mathrm{TP}$ & Recovery \\
\hline 32 & $-3,32$ & 2,23 & $-1,43$ & $-34,41$ & FN & $\mathrm{TP}$ & TP & $\mathrm{TP}$ & TP & TP & TP & TP & TP & Recovery \\
\hline 33 & 4,57 & $-2,28$ & $-0,03$ & $-58,68$ & TN & TN & TN & FP & TN & TN & TN & TN & TN & Death \\
\hline 34 & $-1,84$ & 1,69 & $-2,55$ & $-30,78$ & $\mathrm{TP}$ & TP & ТP & TP & TP & TP & TP & TP & TP & Recovery \\
\hline 35 & $-1,19$ & 2,82 & $-1,16$ & 66,98 & $\mathrm{TP}$ & $\mathrm{TP}$ & TP & TP & $\mathrm{TP}$ & TP & ТP & $\mathrm{TP}$ & $\mathrm{TP}$ & Recovery \\
\hline 36 & 3,09 & $-1,61$ & 1,41 & $-55,05$ & $\mathrm{TN}$ & TN & $\mathrm{TN}$ & $\mathrm{TN}$ & $\mathrm{TN}$ & $\mathrm{TN}$ & $\mathrm{TN}$ & $\mathrm{TN}$ & $\mathrm{TN}$ & Death \\
\hline 37 & $-1,35$ & 0,98 & 2,39 & $-69,83$ & FN & TP & TP & FN & ТP & TP & TP & FN & TP & Recovery \\
\hline 38 & 0,15 & $-0,27$ & 0,56 & $-57,13$ & FN & FP & FN & FN & FN & FN & TP & FN & FN & Recovery \\
\hline 39 & 0,41 & $-1,27$ & 1,89 & $-29,45$ & $\mathrm{TP}$ & FP & FN & FN & FN & FN & FN & FN & FN & Recovery \\
\hline 40 & $-1,56$ & $-0,25$ & $-1,80$ & $-157,32$ & FN & $\mathrm{TP}$ & FN & $\mathrm{TP}$ & TP & TP & ТP & TP & TP & Recovery \\
\hline 41 & $-1,29$ & $-2,54$ & $-0,65$ & 52,08 & $\mathrm{TP}$ & $\mathrm{TP}$ & FN & $\mathrm{TP}$ & TP & TP & FN & $\mathrm{TP}$ & TP & Recovery \\
\hline 42 & 1,29 & $-2,92$ & 3,16 & $-3,81$ & $\mathrm{FP}$ & $\mathrm{TN}$ & $\mathrm{TN}$ & $\mathrm{TN}$ & $\mathrm{TN}$ & $\mathrm{TN}$ & $\mathrm{TN}$ & $\mathrm{TN}$ & $\mathrm{TN}$ & Death \\
\hline 43 & 5,72 & $-1,61$ & 3,35 & $-104,86$ & TN & $\mathrm{TN}$ & $\mathrm{TN}$ & $\mathrm{TN}$ & $\mathrm{TN}$ & $\mathrm{TN}$ & $\mathrm{TN}$ & $\mathrm{TN}$ & $\mathrm{TN}$ & Death \\
\hline 44 & $-0,20$ & 1,45 & 1,08 & 26,036 & $\mathrm{TP}$ & TP & ТP & FN & TP & $\mathrm{FN}$ & ТP & FN & FN & Recovery \\
\hline 45 & $-1,86$ & 0,33 & $-1,47$ & $-142,28$ & FN & TP & $\mathrm{TP}$ & $\mathrm{TP}$ & TP & TP & ТP & $\mathrm{TP}$ & $\mathrm{TP}$ & Recovery \\
\hline 46 & 1,90 & $-1,22$ & 0,07 & 14,15 & FN & FP & FN & FN & FN & FN & FN & FN & FN & Recovery \\
\hline 47 & 0,65 & $-1,92$ & $-1,66$ & 61,02 & $\mathrm{TP}$ & FP & $\mathrm{FN}$ & $\mathrm{TP}$ & $?$ & $\mathrm{FN}$ & $\mathrm{FN}$ & TP & FN & Recovery \\
\hline 48 & $-1,00$ & 3,06 & $-1,49$ & $-36,74$ & $\mathrm{TP}$ & $\mathrm{TP}$ & TP & $\mathrm{TP}$ & TP & TP & ТP & TP & TP & Recovery \\
\hline 49 & 2,58 & $-1,69$ & 2,54 & 222,05 & TN & TN & TN & $\mathrm{TN}$ & $\mathrm{TN}$ & $\mathrm{TN}$ & $\mathrm{TN}$ & TN & $\mathrm{TN}$ & Death \\
\hline
\end{tabular}

\section{Discussion}

Early detection of septic complications, information about the severity of sepsis as well as the ability to predict the outcome of its treatment can have a significant impact on the choice and follow-up of treatment strategy of patients with intra-abdominal infections. Access to such data can be useful in the determination of the urgency of surgical intervention, postoperative monitoring, the necessity of re-operation, and any modifications in antibiotic therapy as well as conservative treatment. Many prognostic methods have been developed in the field of medicine based on parameters which allow for the prediction of prognoses. One of the earliest and most well-known is the Charlson Comorbidity Index (CCI), widely used in patients undergoing dialysis due to renal insufficiency [6]. CCI analyzes parameters similar to the ones proposed in the RPF (age, comorbidities) scale designed by the authors; however, it is based 
on another algorithm. In the case of the Davies Score, age is not included in the derivation of the prognostic prediction [7].

Neither of these prognostic methods which were designed for dialysis patients can be realistically applied to patients with intra-abdominal infections. The ICED scale is used less often compared with the aforementioned ones; it is time-consuming and requires specially-trained personnel in order to analyze all of the necessary parameters [16]. It seems, however, that methods which serve to predict the prognosis in patients operated on due to inflammatory states in the abdominal cavity need to be broadened to include additional biochemical parameters, especially those parameters concerned with the determination of nutritional state and heightened inflammatory response. It has been proven that malnutrition and hipoproteinemia are clearly related with a higher frequency of deaths due to infections and cancer $[17,18]$. The NRI scale has been used to assess the risk of malnutrition, and the incidence and mortality associated with it, in elderly patients [19]. One of the limitations of this prognostic method is the necessity to define the ideal body weight, which is difficult in older and critically ill patients. It has thus become necessary to derive it based on a formula which allows for the theoretical calculation of the ideal body weight. This has led to the development of a newer, more objective prognostic scale: the Geriatric Nutritional Risk Index (GNRI) [20].

In terms of infectious diseases, it is limited in the determination of protein balance as well as age and comorbidities; it is insufficient to predict prognosis of treatment. Among those prognostic methods which take into account inflammatory markers, the Ingenbleek and Carpentier Prognostic Inflammatory and Nutritional Index; (PINI) deserve notable mention [11]. The latter scale analyzes four parameters, two of which are related with malnutrition: CRP and $\alpha 1$ acid glycoprotein (AAG). This method allows the prediction of incidence and mortality in dialysis patients [7,11,21]. PINI has proven to be a valuable indicator of nutritional as well as inflammatory states. In addition, it can be used in the prognosis of trauma, burns, infections [22,23] and recently in cancers [24]. The PINI scale is rather similar to both the proposed RPF scale as well as the AM Risk Calculator $[14,15]$ due to the utilization of two of the three risk factors analyzed: inflammatory state and the determination of malnutrition. It differs primarily by the utilization of two markers: AAG and prealbumin.

In Poland, the AAG marker is not readily available and is not widely used in the clinical setting. However, the pre-albumin level presents itself as very useful in the determination of nutritional insufficiency. This parameter has a permanent place in postoperative monitoring of patients following major visceral surgery. An interesting attempt in the design of an independent indicator of the early prognosis of death in sepsis was proposed by Wunder et al. [25]. The authors of that study analyzed tissue samples taken from 33 patients with sepsis due to various etiologies. They noticed that deviations in the value of PCT and the Acute Physiology and Chronic Health Evaluation (APACHE II) were correlated with a poor prognosis. Similar studies on a larger group of patients with sepsis due to peritonitis or mediastinitis $(n=160)$ were carried out by Nowotny et al. [26]. The latter authors, having utilized both indicators as well as binary logistic regression analysis, were able to identify groups with both a high and a low risk of death. In multifactorial analysis, the results of PCT and APACHE II were determined to be independent, early indicators of mortality in sepsis (SNC=71\%, SPC=77\%) [1].

The prognostic method, RPF scale, proposed by the authors is based on the analysis of seven simple and readily available clinical and biochemical parameters organized into three homogeneous risk factors. It allows for the dichotomic categorization of patients into two groups based on the prediction of two possible prognoses: recovery or death. Grouping together the values calculated for each factor allows for the determination in which patients the course of treatment can be expected to be worse than in others, in the first few hours of hospitalization. Of course, the selection of the appropriate parameters for the prediction of prognosis is a matter of question. Regardless of the wide variety of tissue taken from patients with intra-abdominal infections of various etiologies, the predictive power of the RPF method, with modifications, has proven to be significantly high. The authors believe that the use of multifactorial clinical and biochemical parameters allows for the elimination of errors in the sample group as well as that of unforeseen deviations in the markers chosen in cases involving severe comorbid states.

The sensitivity of the RPF scale was determined to be $79 \%$ and the specificity 95\%; despite the slight degree of uncertainty of two cases which it was impossible to categorize. Two techniques discussed in this article were found to be insufficient in their predictive power. The combined RPF allows for the categorization of all patients by the use of a closely-tailored mathematical technique, but at the cost of a loss in sensitivity (62\%) and specificity (55\%). The prediction determined by the division of the factors by the median seems to be invalid due to the normal distribution of the values of these factors. Typically, in a normal distribution, the mean is considered as the value used to define an index. Thus, there is no justification for the use of the median.

\section{Conclusion}

Upon the conclusion of this investigation, it was determined that the prediction of the treatment outcome of abdominal inflammation is possible based on the statistical analysis of clinical and biochemical parameters and by the use of a factor average prediction engine. However, future studies must consider the slight degree of uncertainty inherent in such predictions.

\section{Acknowledgement}

This study was supported by Medical University of Lodz grant nr. 503/5-138-01/503-51-004

\section{References}

1. Herzog T, Chromik AM, Uhl W (2010) Treatment of complicated intraabdominal infections in the era of multi-drug resistant bacteria. Eur J of Med 1 Researh 15(2): 525-532.

2. Sartelli M, Catena F, Ansalonik L, Leppaniemi A, Taviloglu K, et al. (2012) Complicated intra-abdominal infections in Europe: a comprehensive review of the CIAO study. World J Emerg Surg 7(1): 36. 
3. Moore LJ, McKinley BA, Turner KL, Todd SR, Sucher JF, et al. (2011) The epidemiology of sepsis in general surgery patients. J Trauma 70(3): 672680.

4. Sartelli M, Catena F, Di Saverio S, Ansaloni L, Malangoni M, et al. (2014) Current concept of abdominal sepsis: WSES position paper. World Journal of Emergency Surgery 9: 22.

5. Mokart D, Leone M, Sannini A, Brun JP, Tison A, et al. (2005) Predictive perioperative factors for developing severe sepsis after major surgery. British Journal of Anaesthesia 95(6): 776-781.

6. Charlson ME, P Pompei, KL Ales, CR MacKenzie (1987) A new method of classifying prognostic comorbidity in longitudinal studies: development and validation. Journal of Chronic Diseases 40(5): 373-383.

7. Davies SJ, Russell L, Bryan J, Phillips L, Russell GI (1995) Comorbidity, urea kinetics and appetite in continuous ambulatory peritoneal dialysis patients: their interrelationship and prediction of survival. Am J Kidney Dis 26(2): 353-361.

8. Fried L, J Bernardini, B Piraino (2001) Charlson comorbidity index as a predictor of outcomes in incident peritoneal dialysis patients. American Journal of Kidney Diseases 37(2): 337-342.

9. Buzby GP, LS Knox, LO Crosby, Eisenberg JM, Haakenson CM, et al. (1988) Study protocol: a randomized clinical trial of total parenteral nutrition in malnourished surgical patients. American Journal of Clinical Nutrition 47( 2 suppl): 366-381.

10. Bouillanne O, Morineau G, Dupont C, Coulombel J, Vincent JP, et al. (2005) Geriatric nutritional risk index: a new index for evaluating at-risk elderly medical patients. American Journal of Clinical Nutrition 82(4): 777-783.

11. Ingenbleek Y, Carpentier Y A (1985) A prognostic inflammatory and nutritional index scoring critically ill patients. Int J Vitam Nutr Res 55(1): 91-101.

12. Keats AS (1978) The ASA classification of physical status a recapitulation. Anesthesiology 49(4): 233-236.

13. Le Gall JR, Klar J, Lemeshow S, Saulnier F, Alberti C, et al. (1996) The Logistic Organ Dysfunction system. A new way to assess organ dysfunction in the intensive care unit. ICU Scoring Group. J Am Med Assoc 276(10): 802-810.

14. Jabłoński S, Kozakiewicz M (2012) Proposal for a recovery prediction method for patients affected by acute mediastinitis. World J Emerg Surg 7(1): 11.

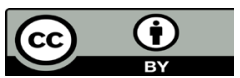

This work is licensed under Creative Commons Attribution 4.0 License

Submission Link: https://biomedres.us/submit-manuscript.php
15. Jabłoński S, Kozakiewicz M (2013) Evaluation of Recovery in Iatrogenic Evoked Acute Mediatinitis Inflammation 36(5): 1055-1063.

16. Wang CHY, Lin YS, Tzao CH, Lee HCH, Huang MH, et al. (2007) Comparison of Charlson comorbidity index and Kaplan-Feinstein index in patients with stage I lung cancer after surgical resection. European Journal of Cardio-Thoracic Surgery 32(6): 877-881.

17. Nuutinen LS, Kauppila A, Ryhanen P, Niinimaki A, Kivinen S, et al. (1982) Intensified nutrition as an adjunct to cytotoxic chemotherapy in gynaecological cancer patients. Clin Oncol 8(2): 107-112.

18. Chen MK, Souba WW, Copeland EM (1991) Nutritional support of the surgical oncology patient. Hematol Oncol Clin North Am 5(1): 125-145.

19. Naber HJ, de Bree A, Schermer TRJ, Bakkeren J, Bär B, et al. (1997) Specificity of indexes of malnutrition when applied to apparently healthy people: the effect of age. Am J Clin Nutr 65(6): 1721-1725.

20. Bouillanne O, Morineau G, Dupont C, Coulombel I, Vincent JP, et al. (2005) Geriatric Nutritional Risk Index: a new index for evaluating atrisk elderly medical patients. Am J Clin Nutr 82(4): 777-783.

21. Perier C, Granouillet R, Chamson A, Gonthier R, Frey J (2002) Nutritional markers, acute phase reactants and tissue inhibitor of matrix metalloproteinase 1 in elderly patients with pressure sores. Gerontology 48(4): 298-301.

22. Gottschlich MM, Baumer T, JenkinsM, Khoury J, Warden GD (1992) The prognostic value of nutritional and inflammatory indices in patients with burns. J Burn Care Rehabil 13(1): 105-113.

23. Bonnefoy M, Ayzac L, Ingenbleek Y, Kostka T, Boisson RC, et al. (1998) Usefulness of the prognostic inflammatory and nutritional index (PINI) in hospitalized elderly patients. Int J Vitam Nutr Res 68(3): 189-195.

24. Walsh D, Mahmoud F, Barna B (2003) Assessment of nutritional status and prognosis in advanced cancer: interleukin-6, C-reactive protein, and the prognostic and inflammatory nutritional index. Support Care Cancer 11(1): 60-62.

25. Wunder C, Eichelbronner O, Roewer N (2004) Are IL-6, IL-10 and PCT plasma concentrations reliable for outcome prediction in severe sepsis? A comparison with APACHE III and SAPS II. Inflamm Res 53(4): 158-163.

26. Novotny A, Emmanuel K, Matevossian E, Kriner M, Ulm K, et al. (2007) Use of procalcitonin for early prediction of lethal outcome of postoperative sepsis. The American Journal of Surgery 194(1): 35-39.

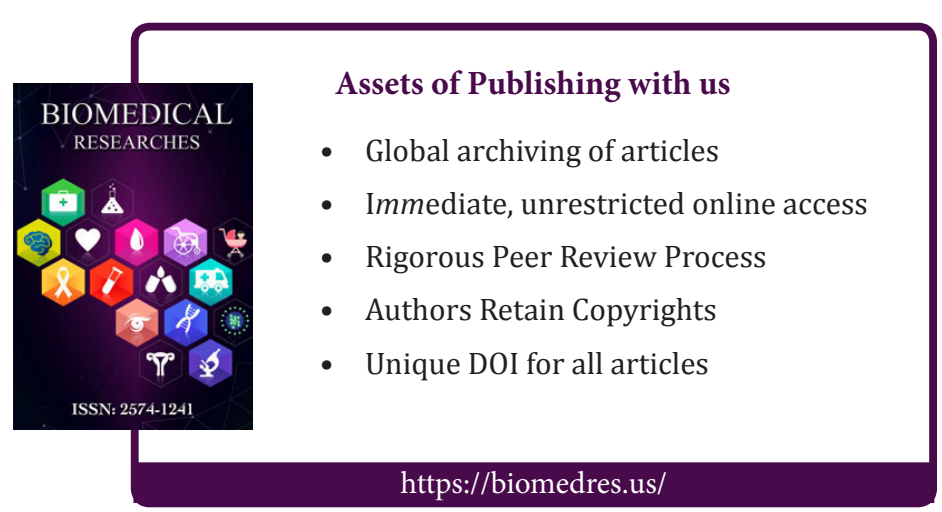

